\title{
Síntomas relacionados con fobia social en personal sanitario
}

\author{
A. J. Madueño Caro, M. Ortega Galán*, E. Benítez Rodríguez**, \\ E. Alcaraz Vera***, F. Forja Pajares*****
}

\begin{abstract}
Espedalista en Mediana Familiar y Comunitaria. Centro de Salud La Laguna. Cádiz. *Diplomada Universitaria en Enfermería. Adjunta Enfermería. Centro de Salud La Laguna. Cádiz. **Especialista en M ediana Preventiva y Salud Pública. Adjunto H ospital Puerta del Mar. Cádiz. ***E specialista en Medicina Familiar y Comunitaria. Coordinador M édico EBAP. Centro de Salud La Laguna. Cádiz. ****Especialista en Mediana Familiar y Comunitaria. Coordinador Médico Distrito Atención Primaria Cádiz-Bahía-Véjer
\end{abstract}

\section{RESUMEN}

Fundamentos: la prevalencia de fobia social en la población general oscila entre el 8-15,6\%, con siderándose un trastorno de ansiedad infradiag nosticada. Se desconoce su prevalencia en perso nal sanitario, quien precisa de realizar actividades de educación a la comunidad y de comunicación científica.

Objetivos: determinar la prevalencia de síntomas relacionados con fobia social en personal sanitario.

Diseño: observacional, descriptivo transversal.

Ámbito de estudio: distrito sanitario de Aten. ción Primaria.

Sujetos: se incluyen como participantes perso nal sanitario (médico y enfermero) con ejercicio en 15 centros de salud.

Mediciones: se solicita cumplimentación, de forma anónima y voluntaria, de un cuestionario elaborado al efecto, escalas de ansiedad social de Liebowitz (LSAS) y de ansiedad/depresión de Goldberg (EADG) al personal médico y de enfer mería trabajadores de 13 centros de salud, en un distrito sanitario de Atención Primaria (población a estudio $=266$ sujetos .

Resultados: grado de respuesta del 39\% $(n=104)$. Se detectó ansiedad social grave en el $1,8 \%$ de los casos (2 individuos), moderada en el $25,7 \%$ (22 individuos) y leve o no patológica en el $76,5 \%$ (80 individuos). Para el conjunto global de profesionales a estudio, se detecta coexistencia entre síntomas relacionados con fobia social y subescalas de la EADG para la ansiedad y depre. sión con significación estadística $(p<0,012$ para
Symptoms in relation with social phobia in health personal

\section{ABSTRACT}

Backgrounds: prevalence of social phobia in general population is between 8-15,6\%. It's unknow prevalence in health personal, who needs to do activities of education to community and cienti fic communications.

Objectives: to determinate the prevalence of symptoms relationated with social phobia in health personal.

Design: transversal study.

Setting: health district of Primare Health Care.

Subjects: personal of fifteen health centers.

Measurements: it's questionated for take anonymous questionary, social phobia of Liebowitz (LSAS) and the anxiety-depression of Golberg (EADG) scales to medical personal and infirmary who were working in a health district (study popu lation= 266 subjects).

Results: answer rate $39 \%(n=104)$. There were severe social anxiety in $1.8 \%$ of all cases (2 sub jects), moderate in $25.7 \%$ (22 subjects) and light or not pathological in $76.5 \%$ (89 subjects). For all professionals included in the study, it is detected the coexistence of symptoms in relation with social phobia and EADG subscales with stadistic signifi cation ( $p<0.0012$ for light anxiety and $p<0.034$ for moderate and severe phobia).

Conclusions: high prevalence of symptoms in relation with social phobia in health personal of a district for a study. It's necessary special atten tion to this pathology and to the evidence of

Aceptación: 11-02-02 
la categoría de ansiedad leve y $p<0,034$ para la categoría de ansiedad de intensidad moderada o severa).

Conclusiones principales: Alta prevalencia de síntomas relacionados con fobia social entre el personal sanitario del distrito a estudio. Es preci sa, una atención especial a esta sintomatología, y a la manifiesta coexistencia de síntomas de ansiedad y/o depresión detectadas.

Palabras clave: Fobia social. Personal sanita rio. Prevalencia. coexistence of anxiety and depression symptoms detected.

Key words: Social phobia. Health personal. Pre valence.

\section{INTRODUCCIÓN}

La definición de fobia social es semejante en los sistemas de clasificación universalmente aceptados (Clasificación Internacional de Enfermedades -CIE 10-), realizada por la Organización Mundial de la Salud (OMS), y la última revisión realizada por la Academia Americana de Psiquiatría (APA) -DSMIV-, resaltando la definición de la CIE 10 que lo característico del trastorno es el miedo marcado a ser el foco de atención o el miedo a comportarse de forma embarazosa o humillante, lo que conduce a la evitación ${ }^{1}$ mientras que la segunda (DSM-IV), destaca el miedo persistente y acusado a situaciones sociales o a actuaciones en público por temor a que resulten embarazosas ${ }^{2}$.

La bibliografía destaca a la fobia social como el trastorno de ansiedad más frecuente, describiéndose en distintos estudios datos de prevalencia que oscilan entre el 7 y el $14 \%$ de la población con edad entre 15 y 54 años $^{3}$.

En otros estudios, que utilizan como instrumento de medida escalas más específicas, como la Flo rence Psychiatric Interview (FPI) se describe una prevalencia del $6,58 \%$ con edad media en los 28,8 años y primeros síntomas a la edad de 15,5 años $^{4}$.

Se describe asociación entre la fobia o ansiedad social y otros trastornos mentales (fundamentalmente ansiedad y/o depresión, aunque también distimia, trastorno obsesivo-compulsivo, abuso de sustancias, otras fobias, etc.) en el $70-80 \%$ de pacientes con el citado trastorno ${ }^{3}$.

Se considera tratamiento de elección para el trastorno de fobia social la terapia cognitiva ${ }^{5-6}$. La intervención farmacológica se propone como alternativa y todos los ensayos clínicos realizados con fármacos, fundamentalmente inhibidores de la recaptación de serotonina ${ }^{7}$ deben compararse a las técnicas comentadas.

El objetivo principal del estudio es determinar la prevalencia de síntomas relacionados con ansiedad o fobia social en la población de personal sanitario (facultativos y enfermería) en un distrito sanitario de Atención Primaria, siendo objetivo secundario conocer la comorbilidad entre síntomas en relación con ansiedad social y síntomas de depresión.

\section{MATERIAL Y MÉTODOS}

El equipo investigador diseña un estudio observacional descriptivo, transversal. El estudio se ubica en el distrito sanitario de Atención Primaria Cádiz-Bahía-Vejer, y se desarrolla durante el mes de enero del 2001. Desde el centro de salud en el que trabaja el equipo investigador se envía correo escrito simultáneo a todos los adjuntos/as de enfermería de los 15 centros de salud que constituyen el distrito sanitario de Atención Primaria, en el que se desarrolla el estudio, siendo la población diana de 266 trabajadores. En el correo que se envía, se justifica el proyecto y se solicita que se distribuya el documento de recogida de datos a la población a estudio. Dicho documento incluye cuestionario elaborado por el equipo investigador y escalas de Liebowitz para ansiedad social (LSAS) (Fig. 1) y escala de ansiedad y depresión de Goldberg (EADG) (Fig. 2) para ansiedad y depresión. Se solicita al personal médico y de enfermería su cumplimentación de forma anónima y voluntaria. Se determina un periodo de tres días naturales para este fin, transcurridos los cuales se recogen por correo los documentos cumplimentados.

El cuestionario elaborado por el equipo investigador reúne variables categóricas relacionadas con la edad, sexo, profesión, antecedentes de haber realizado en el último año o al menos en alguna ocasión sesiones docentes en el centro de trabajo, comunicaciones a foros científicos y/o actividades de educación para la salud a la comunidad.

Como instrumentos de medida de la morbilidad psíquica y de la ansiedad social, utilizamos los descritos a continuación.

La LSAS es un instrumento que recoge 24 situaciones sociales, de ejecución y de interacción social, evaluándose desde una perspectiva de ansie- 


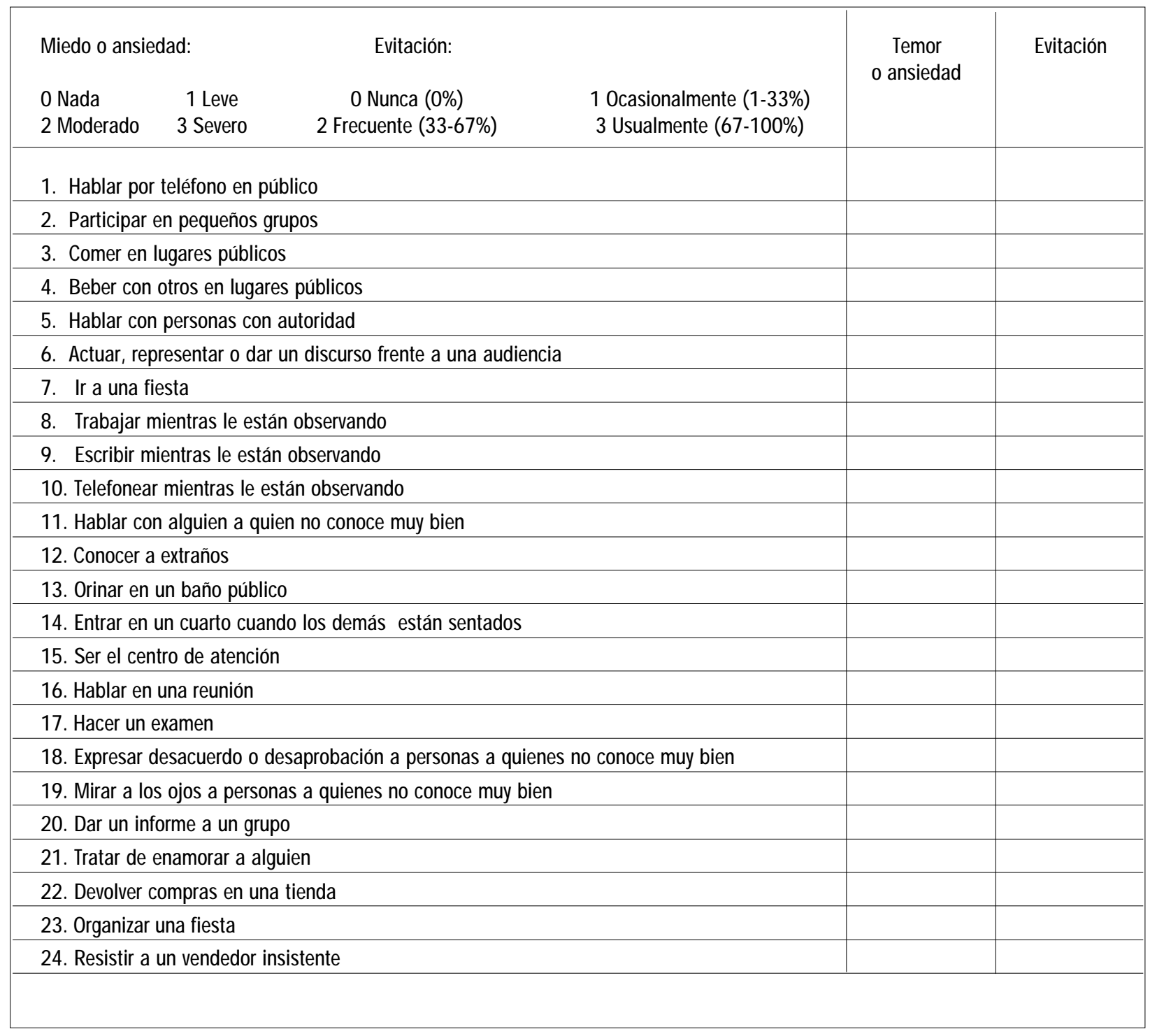

Escala de ansiedad social de Liebowitz (LSAS) (Liebowitz 1987). Tomada de: Bobes J Badía X Luque A Gara 1 M, González M P, Dal-Re R. Validación de las versiones en español de los cuestionarios Liebowitz Social Anxiety Scale, Social Anxiety and Distress Scale y Sheehan Disability Inventory para la evaluación de la fobia social. Medicina Clínica 1999; 112, 14: 530-8.

dad o temor y del grado de evitación. La escala está validada y adaptada al castellano.

La versión en español de la citada escala, junto a otras como la Social Anxiety and Distress Scale $(S A D S)$ y la de Seehan Disability Inventory (SDI), presentan adecuada reproducibilidad (coeficientes de relación intraclase entre 0,63 y 0,88 ), así como consistencia interna (coeficiente 0,72). Los datos relativos a sensibilidad y especificidad de la prueba están contrastados según autores para distintos puntos de corte, siendo posible requerir dicha información ${ }^{8}$.

La medición de intensidad del síntoma ansiedad se realiza utilizando una escala de Likert de cuatro grados, desde $0=$ ninguna ansiedad, a $3=$ ansiedad extrema. Para la evaluación del grado de evitación también se utiliza una escala de Likert de cuatro grados, pero en el caso de la frecuencia: $0=$ nunca, 3 = usualmente (67-100\% de las ocasiones).

Con la LSAS se obtiene una puntuación de ansiedad social total y puntuaciones en subescalas de ansiedad social, ejecución y evitación. En función de la puntuación total se categoriza la intensidad del cuadro clínico de ansiedad en: leve (no patológica), hasta 51 (considerada una categoría correspondiente a la normalidad), moderada, de 52 a 81 y grave, más de $82^{8}$.

La EADG, diseñada para facilitar la detección de malestar emocional; consta de dos subescalas de 9 ítems cada una. Validada en España, ha demostrado 


\author{
Subescala de ansiedad \\ ¿Se ha sentido muy excitado, nervioso o en tensión? \\ ¿Ha estado muy preocupado por algo? \\ ¿Se ha sentido muy irritable? \\ ¿Ha tenido dificultad para relajarse? \\ ¿Ha dormido mal, ha tenido dificultades para dormir? \\ ¿Ha tenido dolores de cabeza o nuca? \\ ¿Ha tenido los siguientes síntomas: temblores, hormigueo, sudores, diarrea? \\ ¿Ha estado preocupado por su salud? \\ ¿Ha tenido alguna dificultad para conciliar el sueño, para quedarse dormido?
}

\section{Subescala de depresión}

¿Se ha sentido con poca energía?

¿Ha perdido usted el interés por las cosas?

¿Ha perdido la confianza en sí mismo?

¿Se ha sentido usted desesperanzado, sin esperanza?

¿Ha tenido dificultades para concentrarse?

¿Ha perdido peso?

¿Se ha estado desperpertando demasiado temprano?

¿Se ha sentido usted enlentecido?

¿Cree usted que ha tenido tendencia a encontrarse peor por las mañanas? su validez para el screening de los procesos psiquiátricos más prevalentes en la Atención Primaria, señalándose como ventajas en su utilización la brevedad y sencillez y poder discriminativo entre ansiedad y depresión, siendo útil como guía para la entrevista clínica. Se aconsejan puntos de corte de mayor rendimiento $2 / 3$ para la subescala de depresión y 3/4 para la subescala de ansiedad. La sensibilidad de la escala es del 83,1\%, especificidad del $81,8 \%$ y su valor predictivo positivo del $95,3 \%$.

En el presente estudio, se establecieron puntos de corte en 2 para la subescala de depresión y en 3 para la de ansiedad.

Los datos obtenidos fueron sometidos a análisis estadístico mediante el programa estadístico EPIInfo 6.0, determinándose descripciones de media, varianza y desviación estándar para variables cuantitativas, de frecuencias absolutas y relativas para variables cualitativas, así como análisis de tabla simple mediante Chi cuadrado para estudio de comorbilidad de síntomas relacionados con fobia social y depresión y/o ansiedad.

\section{RESULTADOS}

Contestaron de forma voluntaria y anónima 104 profesionales $(n=104)$, sobre un total de 266 trabaja- dores en activo en el momento de la recogida de datos de 13 centros de salud del distrito sanitario a estudio ( 2 centros de salud no colaboraron en la realización del estudio por causas desconocidas). Grado de respuesta $=39,1 \%$. La edad media y la distribución para las variables de sexo y profesión fue semejante entre el colectivo que aceptó participar en el estudio y el que declinó hacerlo. La edad media de los individuos incluidos a estudio es de 42 años (DE \pm 7 ). La distribución por sexo fue del $50 \%$ exacto para cada uno ( 52 hombres y 52 mujeres). En cuanto a la profesión de los individuos a estudio, el 46,15\% de los mismos son diplomados universitarios en enfermería (48 DUE), y el resto $(53,85 \%)$, médicos de familia y/o pediatras (56 individuos).

El cuestionario elaborado que se suministró a los sujetos a estudio, recogió variables relativas a la frecuencia en la realización de sesiones clínicas en el centro de trabajo, y/o comunicaciones a foros científicos en el año natural previo al momento de recogida de datos, permitiendo conocer el porcentaje de las citadas actividades realizadas y su distribución por categoría profesional. Así, del colectivo facultativo, el 55,36\% (31 individuos) y el 37,50 \% (18 individuos) del colectivo DUE realizaron al menos una sesión clínica en el año anterior. En cuanto a comunicaciones a foros científicos, el $26,79 \%$ de los facultativos (15 individuos), y el 
$16,67 \%$ ( 8 individuos) de los DUE contestaron haberlas realizado en el año anterior.

Atendiendo al total de individuos incluidos en el estudio, se detectó categoría de ansiedad social grave en el $1,92 \%$ de los casos ( 2 individuos), moderada en el $21,15 \%$ (22 individuos) y leve (no patológica) en el 76,92\% (80 individuos).

Por profesiones, los médicos de familia y/o pediatras que contestaron los cuestionarios y escalas mostraron categoría de ansiedad social leve (no patológica) en el 89,29\% de los casos (50 sujetos), moderada en el 8,93\% (5 sujetos) y categoría de ansiedad social grave en el 1,78\% (1 individuo).

Obteniendo estos resultados atendiendo al sexo, los médicos de sexo masculino mostraron síntomas relacionados con ansiedad social leve en el 96,97\% (32 sujetos) y moderada en el 3,03\% (1 sujeto), mientras que en el sexo femenino, presentaron categoría de ansiedad leve (no patológica) el 78,3\% (18 sujetos), moderada el 17,4\% (4 sujetos) y grave el 4,3\% (1 médico).

En cuanto al personal DUE participante en el estudio, se presentó puntuación correspondiente a una categoría de ansiedad social leve (no patológica) en el $62,5 \%$ (30 individuos), moderada en el $35,42 \%$ (17 individuos) y grave en el $2,08 \%$ (1 sujeto).

Atendiendo a estas variables según su distribución por sexo, los DUE masculinos presentaron niveles de ansiedad leve (no patológica) en el $70 \%$ de los casos (14 sujetos), moderada en el $25 \%$ (5 sujetos) y grave en el $5 \%$ ( 1 sujeto), mientras que los DUE de sexo femenino mostraron ansiedad leve (no patológica) en el 57,14\%, (16 sujetos) moderada en el 42,86\% (12 sujetos), con ningún caso de ansiedad social grave.

El ítem de la escala de Liebowitz que más frecuentemente suscita temor o ansiedad severa entre la población a estudio fue el de "actuar, representar o dar un discurso frente a una audiencia", en un
$24,4 \%$ de los individuos a estudio (25 individuos), seguido del expresado como " hablar en una reunión”, en un 13,46\% (14 individuos).

La escala EADG reveló de forma global para el total de individuos incluidos en el estudio la existencia de ansiedad en el 68,2\% (71 sujetos), y de depresión en el 29,8\% (31 sujetos). Atendiendo a su distribución por sexos, se determinó la presencia de ansiedad en el 69,23\% (36 sujetos) y de depresión en el 25\% (13 sujetos) para el sexo masculino, mientras que para el sexo femenino, apareció ansiedad en el 67,31\% (35 sujetos) y síntomas de depresión en el 34,62\% (18 individuos).

La escala EADG reveló que el 42,42\% (14 individuos) presentaba síntomas de ansiedad y el $15,15 \%$ (5 individuos) de depresión entre los médicos de sexo masculino, mientras que el 69,57\% (16 individuos) y el 43,5\% (10 individuos) de los médicos de sexo femenino presentaban ansiedad y depresión, respectivamente.

Para el conjunto global de profesionales a estudio, se determina coexistencia de síntomas relacionados con fobia social y subescalas de la EADG para la ansiedad y depresión (Tablas I y II).

\section{DISCUSIÓN. CONCLUSIONES}

Los resultados porcentuales descritos sobre la realización de actividades tales como sesiones clínicas y de formación, comunicaciones a foros científicos manifiestamente mejorables, justificaron la consecución del objetivo fundamental del estudio. Los resultados obtenidos sorprenden a los miembros del equipo investigador, dada la alta prevalencia de síntomas relacionados con fobia social entre el personal sanitario del distrito a estudio.

El grado de respuesta obtenido (39\%), puede interpretarse de diferente forma. Por un lado, puede

\begin{tabular}{|c|c|c|c|c|}
\hline \multicolumn{5}{|c|}{ (EN \% Y FRECUENCIA ABSOLUTA) $(p<0,012)$} \\
\hline$n=104$ & \multicolumn{2}{|c|}{\begin{tabular}{cc}
\multicolumn{3}{c}{ EADG } \\
$\begin{array}{cc}\text { Subescala ansiedad + } \\
\%\end{array}$ \\
\end{tabular}} & \multicolumn{2}{|c|}{$\begin{array}{c}\text { EADG } \\
\text { Subescala ansiedad - }\end{array}$} \\
\hline $\begin{array}{l}\text { Ansiedad social } \\
\text { leve (no patológica) }\end{array}$ & 43,3 & 45 & 35,5 & 37 \\
\hline $\begin{array}{l}\text { Ansiedad moderada } 0 \\
\text { grave }\end{array}$ & 18,3 & 19 & 2,8 & 3 \\
\hline \multicolumn{5}{|c|}{$\begin{array}{l}\text { EADG = escala para ansiedad y depresión de Goldberg. } \\
\text { Subescala de ansiedad }+: \text { más de } 3 \text { items afirmativos. } \\
\text { Subescala de ansiedad }-: 3 \text { o menos items afirmativos. }\end{array}$} \\
\hline
\end{tabular}


Tabla II

COEXISTENCIA DE SÍNTOMAS DE FOBIA SOCIAL Y DE DEPRESIÓN (EN \% Y FRECUENCIA ABSOLUTA) $(p<0,034)$

\begin{tabular}{|c|c|c|c|c|}
\hline \multirow[t]{2}{*}{$n=104$} & \multicolumn{2}{|c|}{$\begin{array}{c}\text { EADG } \\
\text { Subescala depresión + }\end{array}$} & \multicolumn{2}{|c|}{$\begin{array}{c}\text { EADG } \\
\text { Subescala depresión - }\end{array}$} \\
\hline & $\%$ & $\mathrm{n}$ & $\%$ & $\mathrm{n}$ \\
\hline $\begin{array}{l}\text { Ansiedad social } \\
\text { leve (no patológica) }\end{array}$ & 17,3 & 18 & 59,6 & 62 \\
\hline $\begin{array}{l}\text { Ansiedad moderada } 0 \\
\text { grave }\end{array}$ & 11,5 & 12 & 11,5 & 12 \\
\hline
\end{tabular}

EADG = escala para ansiedad y depresión de Goldberg.

Subescala de ansiedad + : más de 2 items afirmativos.

Subescala de ansiedad - : 20 menos items afirmativos.

significar que entre los profesionales no participantes se encuentran personas con síntomas fóbicos que reaccionan de forma evitativa a colaborar en el estudio; igualmente, el desconocimiento, desinterés o la poca importancia concedida a esta patología pueden haber influido en el sentido de disminuir el porcentaje de participación. Por otro lado, la alta prevalencia de síntomas podría deberse a una mayor participación de personal sanitario con esta clínica, frente a los que no la padecen.

La búsqueda bibliográfica previa a la realización del estudio (Medline, búsqueda avanzada, utilizando como palabras clave "social phobia", "health personal” y "prevalence”, de forma conjunta y aislada, no halla estudios en la literatura científica cuyos resultados y condiciones metodológicas puedan compararse al realizado en la actualidad, lo que en nuestra opinión, debiera suponer un punto de partida para la realización de ulteriores estudios que puedan describir y analizar datos en relación con la prevalencia de síntomas relacionados con fobia social en personal sanitario.

A pesar de objetivar homogeneidad para variables principales como sexo, edad y profesión existente entre quienes participaron y los que no en el estudio, el equipo investigador asume que el grado de respuesta obtenido puede no contribuir a garantizar la validez de los resultados. No se realizó reenvío de cuestionarios por problema de factibilidad, así como por no existir porcentajes de respuesta en población de estudio similar en otros estudios, aceptándose el obtenido en primera instancia.

Se impone, en cualquier caso, una atención especial a esta sintomatología, y a la manifiesta coexis- tencia de síntomas para la depresión y ansiedad. Actuaciones en este sentido podrían conformarse ofreciendo desde departamentos de salud laboral de los distritos sanitarios en colaboración con los equipos de salud mental existentes en los mismos, programas de terapia cognitiva y asesoramiento psicológico y/o farmacológico a profesionales que identifiquen síntomas que dificulten su ejercicio profesional.

Los resultados deben estimular al profesional médico a identificar de forma temprana estos síntomas, utilizando escalas de fácil aplicación, entre la población demandante de atención sanitaria, sobre todo en quienes ya presenten algún trastorno mental, con especial atención a profesionales que puedan verse invalidados por la existencia de ansiedad o fobia social y sobre el periodo etario adolescente, donde los síntomas fóbicos comienzan a cristalizar, paralelamente a la maduración de la personalidad; así como sugieren formación continuada en el manejo y control de esta entidad.

\section{AGRADECIMIENTOS}

A todo el personal sanitario participante en el estudio

\section{CORRESPONDENCIA:}

Antonio J. Madueño Caro

Plaza Pintor Clemente de Torres $3,7^{\circ} \mathrm{C}$

11010 Cádiz 


\section{Bibliografía}

1. CIE 10. Décima revisión de la Clasificación Internacional de Enfermedades de la Organización Mundial de la Salud. Trastornos mentales y del Comportamiento. Descripciones clínicas y pautas para el diagnóstico. Ginebra: Ed. Meditor, 1992.

2. DSM-IV. Manual de diagnóstico y estadístico de los trastornos mentales. $4^{a}$ ed. Asociación Americana de Psiquiatría. Barcelona: Ed. Masson, 1992.

3. Sareen L, Stein M. A review of the epidemiology and approaches to the treatment of social anxiety disorder. Drugs 2000; 59 (3): 497-509.

4. Faravelli C, Zucchi T, Viviani B, Salmoria R, Perone A, Paionni A, et al. Epidemiology of social phobia: a clinical approach. Eur Psychiatry 2000; 15 (1): 17-24.

5. García Vera, MP, Sanz, Avia MD. La utilización de terapias cognitivas y de la conducta. En: Psiquiatría en Atención Primaria. Madrid: Ed. Aula Médica, 1998.
6. Otto MW. Cognitive-behavioral therapy for social anxiety disorder: model, methods, and outcome. J Clin Psychiatry 1999; 60 (Supl. 9): 14-9.

7. Schatzberg AF. New indications for antidepressants. J Clin Psychiatry 2000; 61 (Supl. 11): 9-17.

8. Bobes J, Badía X, Luque A, García M, González MP, DalRe R. Validación de las versiones en español de los cuestionarios Liebowitz Social Anxiety Scale, Social Anxiety and Distress Scale y Sheehan Disability Inventory para la evaluación de la fobia social. Medicina Clínica 1999; 112, 14 : 530-8.

9. Goldberg D, Hillier VF. A scaled version of the General Health Questionnarie. Psicol Med 1979; 9: 139-45.

10. Montón C, Pérez MJ, Campos R, García Campayo J, Lobo A. Escalas de ansiedad y depresión de Goldberg: una guía de entrevista eficaz para la detección del malestar psíquico. Atención Primaria 1993; 12 (6): 345-9. 